\title{
Kobuviruses carried by Rattus norvegicus in Guangdong, China
}

\author{
Fang-Fei You, Min-Yi Zhang, Huan He, Wen-Qiao He, Yong-Zhi Li and Qing Chen ${ }^{*}$
}

\begin{abstract}
Background: Murine kobuviruses (MuKV) are newly recognized picornaviruses first detected in murine rodents in the USA in 2011. Little information on MuKV epidemiology in murine rodents is available. Therefore, we conducted a survey of the prevalence and genomic characteristics of rat kobuvirus in Guangdong, China.

Results: Fecal samples from 223 rats (Rattus norvegicus) were collected from Guangdong and kobuviruses were detected in $12.6 \%$ (28) of samples. Phylogenetic analysis based on partial 3D and complete VP1 sequence regions showed that rat kobuvirus obtained in this study were genetically closely related to those of rat/mouse kobuvirus reported in other geographical areas. Two near full-length rat kobuvirus genomes (MM33, GZ85) were acquired and phylogenetic analysis of these revealed that they shared very high nucleotide/amino acids identity with one another (95.4\%/99.4\%) and a sewage-derived sequence (86.9\%/93.5\% and 87.5\%/93.7\%, respectively). Comparison with original Aichivirus A strains, such human kobuvirus, revealed amino acid identity values of approximately $80 \%$.
\end{abstract}

Conclusion: Our findings indicate that rat kobuvirus have distinctive genetic characteristics from other Aichivirus $A$ viruses. Additionally, rat kobuvirus may spread via sewage.

Keywords: Kobuvirus, Rattus norvegicus, Prevalence, Genetic characteristic

\section{Background}

Kobuviruses are widespread worldwide and associated with gastroenteritis, respiratory infections, and other clinical symptoms in humans and animals [1]. Kobuvirus was first isolated from a fecal sample from a patient with acute gastroenteritis in Japan, 1989 [2].

The genus Kobuvirus, belongs to family Picornaviridae [3]; its genome includes a 5' untranslated region (UTR); a leader sequence $(\mathrm{L})$; a large open frame encoding a single polyprotein of approximately 2400-2500 amino acids, which cleaves to produce three viral structural proteins (VP0, VP1, and VP3) and seven non-structural proteins (2A-2C and 3A-3D); a 3'UTR; and a poly (A) tail [4]. The $3 \mathrm{D}$ segment is a conserved region, while the VP1 protein is the most variable [5]. The genus

\footnotetext{
*Correspondence: qch.2009@163.com

Department of Epidemiology, School of Public Health, Guangdong Provincial Key Laboratory of Tropical Disease Research, Southern Medical University, 1838 North Road Guangzhou, Guangzhou 510515, China
}

Kobuvirus consists of six species, including AichivirusA (AiVA; formerly Aichi virus), Aichivirus B (AiV B; formerly Bovine kobuvirus), Aichivirus $C$ (AiV C; formerly Porcine kobuvirus), Aichivirus $D$ (AiV D; formerly Kagovirus 1), Aichivirus E (AiV E; formerly rabbit kobuvirus), and Aichivirus $F$ (AiV F; formerly bat kobuvirus) [6]. Human kobuvirus, canine kobuvirus, feline kobuvirus, and murine kobuvirus (MuKV) belong to AiV A [7].

Kobuviruses have a wide host range worldwide, including humans, dogs, cats, pigs, cattle, goats, sheep, bats, rats, and mice [1, 4, 8-10]. The mouse kobuvirus was detected in Peromyscus crinitus and Peromyscus maniculatus mice in the USA in 2011 [11]. MuKV have since been detected in fecal samples from several countries, including Vietnam [6], Hungary [5], and the USA [12]. The study conducted in Vietnam showed that kobuviruses can be present in different rat species, including Rattus argentiventer, Rattus losea, and Rattus norvegicus 
[6]. Complete rat kobuvirus sequences from Rattus norvegicus were reported in a study conducted in Hungary [13]; however, information about Murine kobuviruses remains scarce.

The Norway rat (Rattus norvegicus) is the largest commensal rodent worldwide. They always reside near human activity and are therefore a concern for public health. There are no reports describing Kobuvirus prevalence and genetic characteristics in Rattus norvegicus in China. Here, we report the presence and genetic characteristics of rat kobuvirus in Rattus norvegicus in Guangdong, a province of southern China.

\section{Results}

\section{Prevalence of rat kobuvirus}

A total of 223 Rattus norvegicus animals were trapped. Twenty-eight (12.6\%) Rattus norvegicus fecal samples were positive for Kobuvirus (3D region, 216nucleotides (nt)). Of samples from Guangzhou city, 26/135 (19.3\%) were positive, while the rate was $2 / 88$ (2.2\%) among samples from Maoming city.

\section{Phylogenetic analysis}

Phylogenetic trees, including representative sequences detected in this study and reference sequences from Genbank, were constructed based on partial 3D sequences (461 nt) (Fig. 1). Sequences isolated from the two cities were highly conserved with one another, with nucleotide sequence identities of 92.6 to $99.8 \%$. Further, the sequences identified in this study clustered within the Aichivirus A group, which also includes human kobuvirus, rat/mouse kobuvirus, canine kobuvirus, and feline kobuvirus.

Complete VP1 sequences were further generated from samples positive for the partial 3D gene using a one-step PCR. Sequences from several representative isolates generated in this study, along with VP1 sequences from Kobuvirus strains available in GenBank were used to construct a phylogenetic tree (Fig. 2). The VP1 sequences of rat kobuviruses identified in this study shared $82.1-98.6 \%$ nucleotide and 85.099.0\% amino acid identities, as determined using DNASTAR software. Phylogenetic analysis based on VP1 nucleotide sequences showed that these sequences and two reference rat kobuvirus sequences were clustered together. Additionally, a bat kobuvirus (MF947381) [6] clustered together with those sequences rat-derived. DNASTAR software was further used to analyze the homology alignment among sequences, and the results suggested that a bat kobuvirus sequence (MF947381), derived from Vietnam, had highly similarity to the rat kobuvirus sequences detected in this study, with nucleotide (amino acids) identity values of $71.4 \%(75.3 \%)$ to $76.8 \%$ (84.1\%); however, our sequences showed lower similarity to other bat kobuviruses reported.

\section{Near-complete rat kobuvirus genome sequences}

Two almost full-length rat kobuvirus genomes (GZ85 and MM33; 7750 and $7734 \mathrm{nt}$, respectively) were obtained from two rat samples. A phylogenetic tree was constructed based on Kobuvirus $A-F$ genome sequences from GenBank and these two kobuviruses (Fig. 3). Both the MM33 and GZ85 genomes showed highest nucleotide (93.44 and 93.77\%) and amino acid (98.63 and 98.90\%) identities to a rat kobuvirus, Aichivirus A, strain Wencheng-Rt386-2 (China) (MF352432.1). Further, sewage-derived kobuvirus (JQ898342.1) in Nepal and the two rat kobuviruses grouped together, with the sewage-derived sequence showing 86.9 and $87.4 \%$ nucleotide and 93.5 and $93.7 \%$ amino acid identities to the genomes detected in this study (MM33 and GZ85, respectively). Comparisons between the full sequences and L/P1/P2/P3 fragments of MM33 and reference Kobuvirus sequences from several other species and the environment are shown in Table 1 . The MM33 genome shared a relatively high nucleotide/amino acid identity with human kobuvirus (77.4\%/81.49\%) and rat kobuvirus $(92.8 \% / 98.3 \%)$, respectively, while it had a low nucleotide homology with bovine $(59.1 \%)$ and swine $(59.3 \%)$ kobuviruses. In the P1, P2, and P3 regions, MM33 kobuvirus showed more than $70 \%$ nucleotide and $80 \%$ amino acid identity with canine, feline, rat, and human kobuviruses, which belong to the Aichivirus A. Further, the MM33 sequence shared $>90 \%$ amino acid sequence identity in the P1, P2, and P3 regions with the sample from untreated sewage.

\section{Similarity plot analysis}

Similarity plot analysis was conducted using SimPlot 3.5.1 to characterize the MM33 and GZ85 sequences. Standard similarity plot analysis, with the Aichivirus $A$ strain Wencheng-Rt386-2 polyprotein gene (MF352432.1) as the query, showed that MM33 and GZ85 exhibited relatively high similarity to the query sequence in the 5 'UTR, VP3, $2 \mathrm{~A}-2 \mathrm{C}, 3 \mathrm{~B}-3 \mathrm{C}$, and $3 \mathrm{D}$ genome regions, with lower levels of similarity in the L, VP0, VP1, 3A regions, and the sequence connecting $3 \mathrm{C}$ and $3 \mathrm{D}$ (Fig. 4).

\section{Discussion}

To our best knowledge, this is the first study to determine the prevalence and conduct genome analysis of rat kobuvirus from Rattus norvegicus in China. We found a mean prevalence of Kobuvirus in Rattus norvegicus of $12.6 \%$. The animals were captured from two cities; the prevalence of Kobuvirus in Guangzhou was 19.3\%, while 


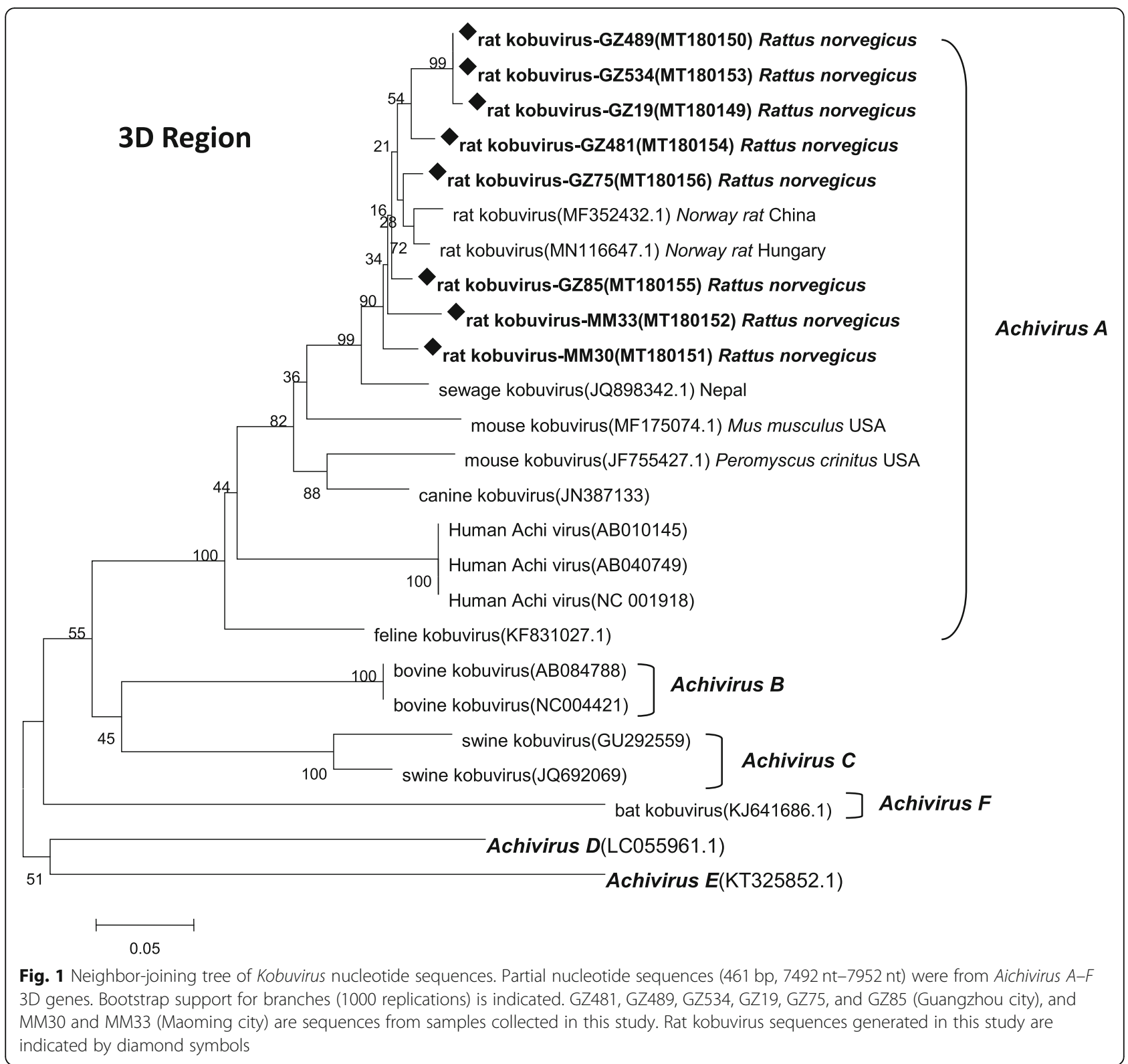

that in Maoming was 2.2\%. Rat kobuvirus were detected in $50 \%$ of Rattus norvegicus fecal samples in New York in 2014 and Kobuvirus are present in approximately 50\% of free-living and laboratory rats (Rattus norvegicus) in Hungary [13, 14], while in Vietnam, the prevalence of Kobuvirus in rats was 17\% [6]. In New York (Manhattan and Queens sites) in 2018, mouse kobuvirus was detected in $17.5 \%$ of Mus musculus samples. These observed variations in prevalence may be attributable to differences in rodent habitat environments. In general, our data support that Kobuvirus is common in rats and warrants research attention.

Phylogenetic trees based on partial 3D or VP1 regions showed that rat kobuvirus sequences cluster closely together in a clade, regardless of originating from different geographical areas. This suggests that rat kobuvirus has distinctive characteristics that differ from other Aichivirus $A$ viruses. Interestingly, we found that a bat kobuvirus from Vietnam (MF947381) [6] shared high nucleotide/amino acids identity (71.4/ $75.3 \%-76.8 / 84.1 \%$ ) with our rat-derived sequences. In addition, in another study from our laboratory, we found that part (461 nt) of Kobuvirus nucleotide sequences (MT180166) detected in bat feces in Guangdong showed high identity to rat kobuvirus (93.5-99.3\%). These data suggest that there may be interaction between bats and rats in the environment, and offer the possibility that the viruses may have 


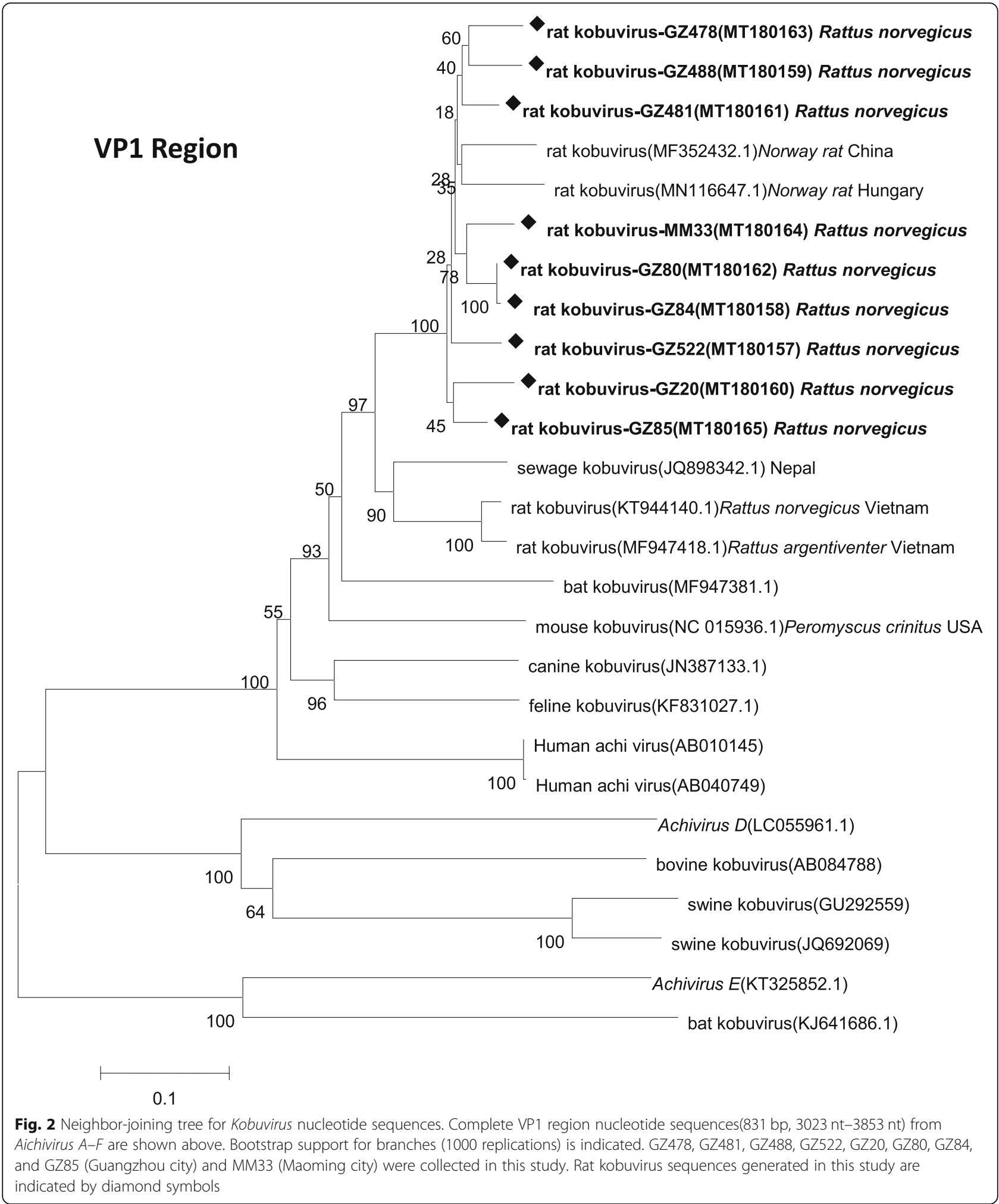

spread among different species. According to ancestral reconstruction analysis, Kobuviruses are likely to jump from rodents to bats [6]. A similar situation also occurs between bovine and swine species, with a previous finding suggesting that interspecies transmission between porcine-bovine kobuviruses may have 


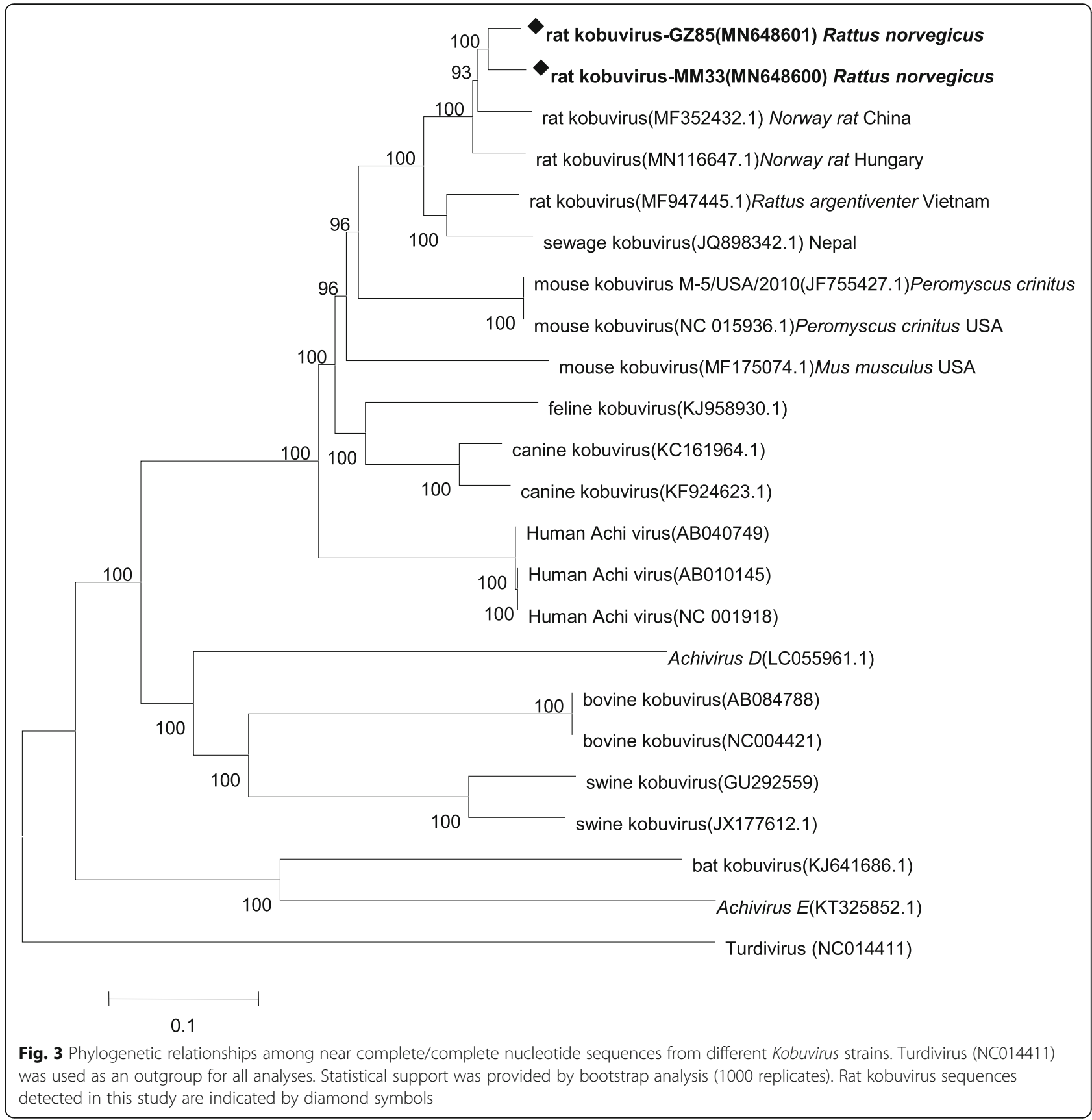

occurred in nature [15]; however, there remains a lack of evidence that Kobuvirus can transmit from murine rodents to bats, or from bats to murine rodents. Hence, more evidence is required before crossspecies transmission in Kobuvirus can be proven.

The near-complete sequences identified in this study shared more than $85 \%$ nucleotide and $90 \%$ amino acid identity to a strain from untreated sewage, similar to a report from New York [14]. These data suggest that Kobuvirus may transmit via water in the environment [16]. Comparison of the full sequence, MM33 with other full reference sequences revealed that MM33 showed the highest nucleotide/amino acid identity to rat kobuvirus $(92.8 \% / 98.3 \%)$, with identity values $<60 \%$ to cattle and pig sequences. Similarity plot analysis showed that MM33 and GZ85 had lower similarities at the L, VP0, VP1, and 3A loci as well as a region near the start of the $3 \mathrm{D}$, indicating possible changes in these regions. These data increase knowledge of murine kobuvirus evolution. 
Table 1 Putative nucleotide/amino acid identity of the rat kobuvirus (MM33) with reference Kobuvirus strains ${ }^{\text {a }}$

\begin{tabular}{|c|c|c|c|c|c|c|c|}
\hline \multirow[t]{2}{*}{ Gene region } & \multicolumn{6}{|c|}{ Nucleotide identity (\%)/amino acid identity (\%) } & \multirow{2}{*}{$\begin{array}{l}\text { Untreated } \\
\text { sewage }\end{array}$} \\
\hline & Human & Bovine & Swine & Feline & Canine & Rat & \\
\hline$\overline{L^{b}}$ & $67.3 / 61.05$ & $44.1 / 39.26$ & $43.1 / 35.0$ & $62.0 / 46.82$ & $60.6 / 56.39$ & $85.4 / 90.18$ & - \\
\hline$P 1^{b}$ & $75.1 / 79.76$ & $57.7 / 52.96$ & $59.6 / 55.27$ & $74.8 / 80.58$ & $75.5 / 77.28$ & $91.7 / 99.08$ & $84.6 / 93.29$ \\
\hline$P 2^{b}$ & $77.9 / 83.11$ & $63.2 / 62.52$ & $62.2 / 59.44$ & $77.9 / 84.75$ & $79.7 / 85.57$ & $93.7 / 98.52$ & $87.5 / 93.93$ \\
\hline$P 3^{b}$ & $80.3 / 87.0$ & 64.9/63.59 & $63.7 / 64.7$ & $80.2 / 85.54$ & 82.3/86.6 & $94.1 / 98.94$ & $87.8 / 93.10$ \\
\hline Full sequence & $77.4 / 81.49$ & $58.9 / 57.2$ & $59.5 / 57.22$ & $76.7 / 81.26$ & $77.7 / 80.67$ & $92.8 / 98.3$ & $84.9 / 93.48$ \\
\hline
\end{tabular}

${ }^{a}$ Reference Kobuvirus strains include: human (accession no. AB040749), bovine (accession no. AB084788.1), canine (accession no. JQ911763.1), rat (accession no. MN116647.1), swine (accession no.EU787450.2), feline (accession no. KJ958930.1), and untreated sewage (accession no. JQ898342.1)

b L: Kobuvirus genome leader sequence

(Human kobuvirus:745-1253 nt, Bovine kobuvirus:809-1368 nt, Swine kobuvirus:577-1160 nt, Feline kobuvirus:718-1221 nt, Canine kobuvirus:713-1225 nt, Rat kobuvirus: 737-1228 $\mathrm{nt}$, Sewage kobuvirus:not mentioned)

P1: structural viral protein, including the VP0, VP1, and VP3 regions

(Human kobuvirus:1254-3791 nt, Bovine kobuvirus:1369-3939 nt, Swine kobuvirus:1161-3689 nt, Feline kobuvirus:1222-3780 nt, Canine kobuvirus:1226-3874 nt, Rat kobuvirus:1229-3853 nt, Sewage kobuvirus:181-2421 nt)

P2: nonstructural protein, including regions $2 \mathrm{~A}-2 \mathrm{C}$

(Human kobuvirus:3792-5699 nt, Bovine kobuvirus:3940-5841 nt, Swine kobuvirus:3690-5687 nt, Feline kobuvirus:3781-5688 nt, Canine kobuvirus:3875-5706 nt, Rat kobuvirus:3854-5686 nt, Sewage kobuvirus:2422-4329 nt)

P3: nonstructural protein, including regions 3A-3D

(Human kobuvirus:5700-8043 nt, Bovine kobuvirus:5842-8200 nt, Swine kobuvirus:5688-8043 nt, Feline kobuvirus:5689-8029 nt, Canine kobuvirus:5707-8048 nt, Rat kobuvirus:5687-8030 nt, Sewage kobuvirus:4330-6672 nt)

\section{Conclusion}

This is the first epidemiological study of rat kobuvirus in Rattus norvegicus in China, and demonstrates that rat kobuviruses are common in Rattus norvegicus feces. Rat kobuvirus have distinctive genetic characteristics that differ from other original Aichivirus A. Additionally, rat kobuvirus may spread via sewage. Several Kobuvirus nucleotide sequences detected from bat feces showed high similarity to rat kobuvirus, suggesting that interspecies transmission between murine rodents and bats may be possible. More evidence to support these findings should be sought.

\section{Methods \\ Samples}

The rats were captured close to human residences using cage traps, between January 2016 and July 2017 in two cities, Guangzhou and Maoming, about $350 \mathrm{~km}$ from one another in Guangdong Province. The rats were anaesthetized with diethyl ether. Species of trapped animals were determined by sequencing the cytochrome B (cytB) gene [17]. Fecal samples (approximately $0.2 \mathrm{~g}$ per sample) were obtained from each animal, then immersed in $700 \mu$ l phosphate-buffered saline

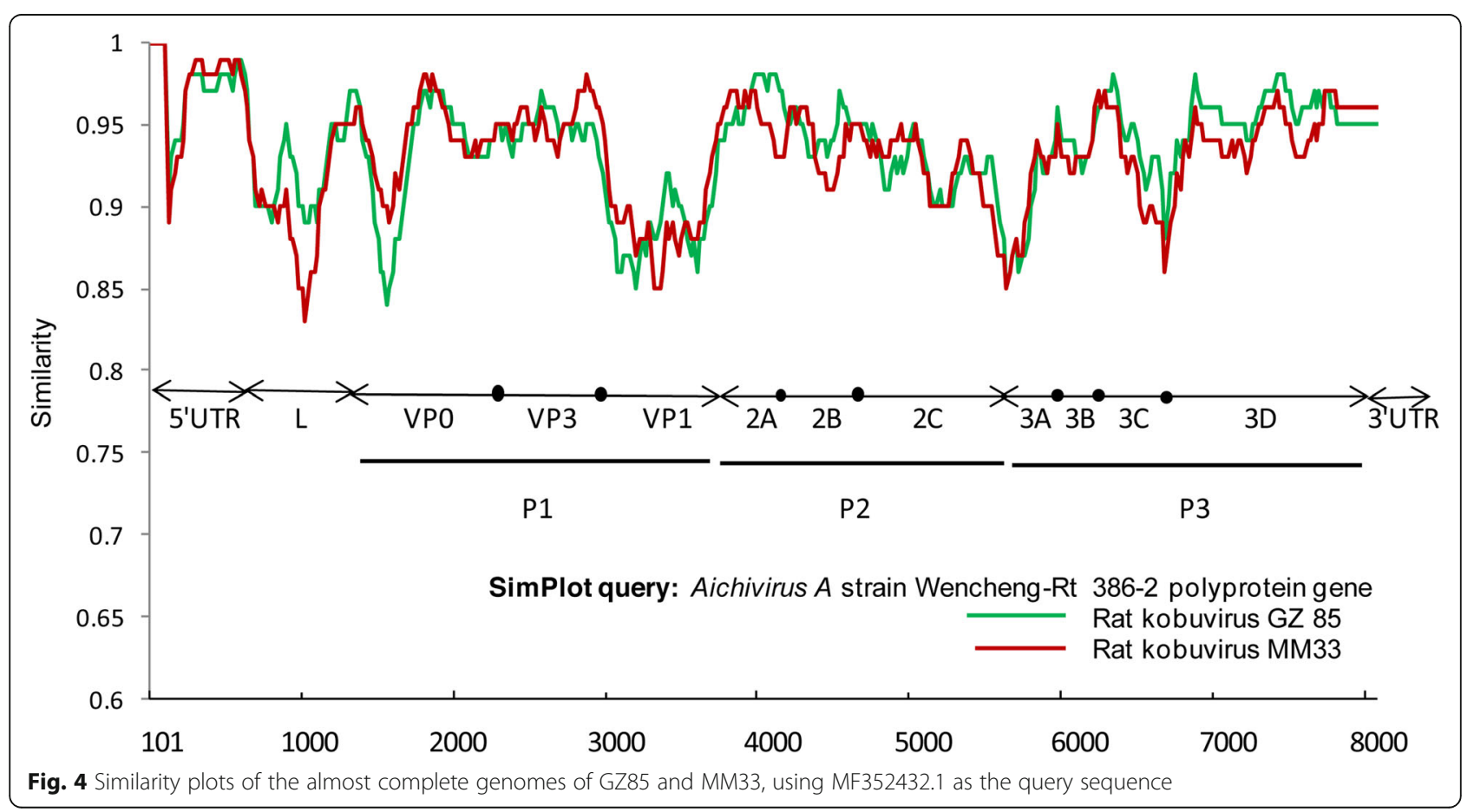


(PBS) $\left(0.03 \%\right.$ homogenate), and stored at $-80{ }^{\circ} \mathrm{C}$. After collecting stool samples, the rats were used for other studies.

\section{Nucleic acid extraction and RT-PCR detection}

RNA and DNA were extracted from the supernatants of fecal samples using the MiniBEST Viral RNA/DNA Extraction Kit, according to the manufacturer's instructions (TaKaRa, Kusatsu, Japan). RT-PCR was conducted using a pair of previously described primers (UNIV-kobu-F and R) to amplify a 216-nt fragment located in the conserved 3D region, encoding the RNA-dependent RNA polymerase [18]. To amplify a longer 3D region, nucleotide primer sequences were designed as follows: 3D-F (5'-CTCCGGTTGTGTGSCCACTTCC-3'; 7492 nt) and 3D-R (5'-AGCACTGCTCGCGCACTTTCAT-3'; 7952 nt), to amplify a $461 \mathrm{bp}$ region. For $3 \mathrm{D}$ gene-positive samples, a 831 bp region of the VP1 gene was amplified by PCR using the VP1-F/VP1-R primer pair [13]. All PCR conditions were $94{ }^{\circ} \mathrm{C}, 3 \mathrm{~min} ; 94^{\circ} \mathrm{C}, 30$ s, $56^{\circ} \mathrm{C}, 1.5$ min, $72{ }^{\circ} \mathrm{C}, 1 \mathrm{~min}, 40$ cycles; $72{ }^{\circ} \mathrm{C}, 10 \mathrm{~min}$. Amplicons were analyzed on $1 \%$ agarose gels followed by UV light trans-illumination.

\section{Full viral genome sequencing}

To amplify full viral genomes, 17 pairs of primers were designed according to the GenBank reference sequence (accession number: MF352432.1) (data not shown). Lasergene SeqMan software (DNASTAR, Inc. USA, Wisconsin, Madison) was used for sequence assembly. Finally, two almost full-length rat kobuvirus genomes were obtained (GenBank accession numbers: MN648600. MN648601).

\section{Phylogenetic analyses}

Phylogenetic analysis of Kobuviruses was performed using the Neighbor-joining method in MEGA v.6.0 (Oxford Molecular Ltd., UK). Multiple alignments were performed using the Clustal W multiple sequence alignment program in MEGA 6.0. Sequences were aligned for homology using DNASTAR software. Similarity plot analysis was conducted using SimPlot 3.5.1.

\section{Abbreviations \\ MuKV: Murine kobuviruses; AiVA/B/C/D/E/F: Aichivirus A/B/C/D/E/F; CytB: cytochrome B}

\section{Acknowledgements}

We deeply appreciate the contribution to this paper made by my teammates concerning the sample collection and technical assistance.

\section{Authors' contributions}

QC designed the study and revised the manuscript. FFY wrote the article and completed the experiment. MYZ, HH, WQH and YZL provided technical assistance. All authors read and approved the final manuscript.

\section{Funding}

This work was supported by the National Natural Science Foundation of China (grant No. 81973107)

The funding body had no role in study design, sample collection, analysis,interpretation of data, writing the manuscript and decision to publish.

Availability of data and materials

All data generated or analyzed during this study are included in this published article. Access to raw data can be acquired by connecting to the corresponding author via email.

\section{Ethics approval and consent to participate}

The study protocol was approved by the Animal Ethics and Welfare Committee of the School of Public Health, Southern Medical University and adhered to the guidelines for the Rules for the Implementation of Laboratory Animal Medicine (1998) from the Ministry of Health, China. All surgical procedures were performed under anesthesia in efforts to minimize suffering. Endangered or protected species were not involved in this study.

\section{Consent for publication}

Not applicable.

\section{Competing interests}

The authors declare no potential conflicts of interest with respect to the research, authorship, and/or publication of this article.

Received: 7 February 2020 Accepted: 27 March 2020

Published online: 15 April 2020

\section{References}

1. Khamrin P, Maneekarn N, Okitsu S, Ushijima H. Epidemiology of human and animal kobuviruses. Virusdisease. 2014;25(2):195-200.

2. Yamashita T, Kobayashi S, Sakae K, Nakata S, Chiba S, Ishihara Y, Isomura S. Isolation of cytopathic small round viruses with BS-C-1 cells from patients with gastroenteritis. J Infect Dis. 1991;164(5):954-7.

3. Adams MJ, King AM, Carstens EB. Ratification vote on taxonomic proposals to the international committee on taxonomy of viruses (2013). Arch Virol. 2013;158(9):2023-30

4. Akagami M, Ito M, Niira K, Kuroda M, Masuda T, Haga K, Tsuchiaka S, Naoi Y, Kishimoto M, Sano K, et al. Complete genome analysis of porcine kobuviruses from the feces of pigs in Japan. Virus Genes. 2017;53(4):593602.

5. Reuter G, Boros A, Pankovics P. Kobuviruses - a comprehensive review. Rev Med Virol. 2011;21(1):32-41.

6. Lu L, Van Dung N, Ivens A, Bogaardt C. Genetic diversity and cross-species transmission of kobuviruses in Vietnam. Virus Evol. 2018:4(1):vey002.

7. Nantel-Fortier N, Lachapelle V, Letellier A, L'Homme Y, Brassard J. Kobuvirus shedding dynamics in a swine production system and their association with diarrhea. Vet Microbiol. 2019;235:319-26.

8. Li H, Tang $\mathrm{C}$, Yue H. Molecular detection and genomic characteristics of bovine kobuvirus from dairy calves in China. Infect, Genet Evol. 2019;74: 103939

9. Charoenkul K, Janetanakit T, Chaiyawong S, Bunpapong N, Boonyapisitsopa $S$, Tangwangvivat $R$, Amonsin A: First detection and genetic characterization of canine Kobuvirus in domestic dogs in Thailand. BMC Vet Res. 2019;15(1): 254.

10. Bergallo M, Galliano I, Montanari P, Rassu M, Dapra V. Aichivirus in children with diarrhea in northern Italy. Intervirology. 2017;60(5):196-200.

11. Phan TG, Kapusinszky B, Wang C, Rose RK, Lipton HL, Delwart EL. The fecal viral flora of wild rodents. PLoS Pathog. 2011;7(9):e1002218.

12. Williams SH, Che X, Garcia JA, Klena JD, Lee B, Muller D, Ulrich W, Corrigan RM, Nichol S, Jain K et al. Viral Diversity of House Mice in New York City. mBio. 2018;9(2):e01354-17.

13. Boros A, Orlovacz K, Pankovics P, Szekeres S, Foldvari G, Fahsbender E, Delwart $E$, Reuter $G$. Diverse picornaviruses are prevalent among free-living and laboratory rats (Rattus norvegicus) in Hungary and can cause disseminated infections. Infect Genet Evol. 2019;75:103988.

14. Firth $\mathrm{C}$, Bhat M, Firth MA, Williams SH, Frye MJ, Simmonds $\mathrm{P}$, Conte JM, Ng J, Garcia J, Bhuva NP et al: Detection of zoonotic pathogens and 
characterization of novel viruses carried by commensal Rattus norvegicus in New York City mBio 2014, 5(5):e01933-e01914..

15. Khamrin P, Maneekarn N, Hidaka S, Kishikawa S, Ushijima K, Okitsu S,

Ushijima H. Molecular detection of kobuvirus sequences in stool samples collected from healthy pigs in Japan. Infect Genet Evol. 2010;10(7):950-4.

16. Ng TF, Marine R, Wang C, Simmonds P, Kapusinszky B, Bodhidatta L, Oderinde BS, Wommack KE, Delwart E. High variety of known and new RNA and DNA viruses of diverse origins in untreated sewage. J Virol. 2012;86(22): 12161-75.

17. Schlegel M, Ali HS, Stieger N, Groschup MH, Wolf R, Ulrich RG. Molecular identification of small mammal species using novel cytochrome B genederived degenerated primers. Biochem Genet. 2012;50(5-6):440-7.

18. Reuter G, Boldizsar A, Pankovics P. Complete nucleotide and amino acid sequences and genetic organization of porcine kobuvirus, a member of a new species in the genus Kobuvirus, family Picornaviridae. Arch Virol. 2009; 154(1):101-8.

\section{Publisher's Note}

Springer Nature remains neutral with regard to jurisdictional claims in published maps and institutional affiliations.

Ready to submit your research? Choose BMC and benefit from:

- fast, convenient online submission

- thorough peer review by experienced researchers in your field

- rapid publication on acceptance

- support for research data, including large and complex data types

- gold Open Access which fosters wider collaboration and increased citations

- maximum visibility for your research: over $100 \mathrm{M}$ website views per year

At BMC, research is always in progress.

Learn more biomedcentral.com/submissions 